\title{
МЕТОД ОПТИМИЗАЦИИ ТОПЛИВНО-ЭНЕРГЕТИЧЕСКОГО БАЛАНСА (ТЭБ) РАЙОНА
}

.K. JAANIMAGI. RAJOONI KUTUSE-ENERGIABILANSI OPTIMEERIMISE MEETOD

K. JAANIMAGI. OPTIMIZATION METHOD OF THE REGIONAL FUEL ENERGY BALANCE

\author{
(Представлена И. Эпиком)
}

Исследование ТЭБ района начинается с определения и упорядочения целей оптимизации. Главными целями функционирующего топливноэнергетического комплекса (ТЭК) являются экономически целесообразное расходование имеющихся ресурсов, надежное обеспечение ими потребителей и сведение до минимума вредного воздействия ТЭК на другие системы и окружающую среду. Многоцелевой подход предполагает сравнение различных сочетаний допустимых решений и выбор из них экономически наиболее эффективного. С этой целью вводится система показателей, количественно определяющих цель. Допустимые решения удовлетворяют ограничениям, отвечающим нормативным характеристикам района ТЭБ.

Исходная математическая модель ТЭБ района имеет вид

$$
\begin{aligned}
& f_{1}(\bar{x})=E\left(\sum_{i=1}^{n} \sum_{l=1}^{m} c_{i l} x_{i l}\right) \rightarrow \min , \\
& f_{2}^{k}(\bar{x})=E\left(\sum_{i=1}^{n} \sum_{l=1}^{m} a_{i l}^{k} x_{i l}\right) \rightarrow \min , \\
& f_{3}(\bar{x})=E\left(\sum_{i=1}^{n} \sum_{l=1}^{m} \mathrm{u}_{i l} x_{i l}\right) \rightarrow \min , \\
& f_{4}(\bar{x})=E\left(\sum_{i=1}^{n} \sum_{l=1}^{m} q_{i l} x_{i l}\right) \rightarrow \min
\end{aligned}
$$

при $\bar{x}=\left\{x_{i l}\right\}, i=\overline{1, n}, l=\overline{1, m}$, принадлежащем области $D$, задаваемой неравенствами

$$
\begin{aligned}
& P\left(\sum_{i=1}^{n} \eta_{i l} x_{i l} \geqslant Q_{l}\right) \geqslant \alpha_{l}, \\
& P\left(\sum_{i=1}^{n} a_{i l}^{k} x_{i l} \leqslant Y_{l}^{k}\right) \geqslant \gamma_{l}^{k}, \quad k=\overline{1, K},
\end{aligned}
$$




$$
\sum_{l=1}^{m} x_{i l} \leqslant x_{i} .
$$

Здесь $f_{1}(\bar{x})$ - суммарные приведенные затраты ТЭК, $f_{2}{ }^{k}(\bar{x})$ - суммарные выбросы $k$-го загрязняющего вещества в район ТЭК, $f_{3}(\bar{x})-$ суммарные людские ресурсы ТЭК, $f_{4}(\bar{x})$ - техническая ненадежность ТЭК, $q_{i l}$ - вероятность выхода установки $l$-го потребителя при сжигании $i$-го топлива. Остальные параметры и переменные задачи (1)-(7) описаны в $\left[{ }^{1}\right]$.

Условие (6), вводится для случая превышения предельно допустимых выбросов отдельных потребителей при общем благополучном состоянии окружающей среды в районе. Согласно методу, изложенному в $\left[{ }^{2}\right]$, область $(5)-(7)$ может быть описана системой детерминированных неравенств. Решение задачи (1)-(7) включает в себя шесть этапов:

1. Сведение критериев $f_{1}-f_{4}$ к безразмерным величинам.

2. Нахождение вектора идеальных решений $f^{\text {ид }}=\left(f_{1}^{\text {ид }}, f_{2}^{\text {ид }}, f_{3}^{\text {ид }}, f_{4}^{\text {ид }}\right)$, где $f_{j}^{\text {ид }}\left(j=\overline{1,4)}\right.$ - оптимадьное значение целевой функции $f_{j}$ на области $(5)-(7)$.

3. Получение $m \geqslant 4$ допустимых решений $\bar{x}_{1}, \bar{x}_{2}, \bar{x}_{3}, \ldots, \bar{x}_{m}$.

4. Группировка их по признаку предпочтительности: $\bar{x}_{1}$ предпочтительнее $\left(\{) \bar{x}_{2}\right.$, если оно наименее удалено от идеального решения. Пусть $\bar{x}_{1}\left\{\bar{x}_{2}\left\{\ldots\left\{\bar{x}_{m}\right.\right.\right.$.

5. Нахождение весовых коэффициентов $\lambda_{j}$ таких, что $\Phi(\bar{x})=$ $=\sum_{j=1}^{4} \lambda_{j} f_{j}(\bar{x}) ; \sum_{j=1}^{4} \lambda_{j}=1$, исходя из принципа аддитивной полезности (отметим, что для этой цели пригоден и алгоритм метода нิаименьших квадратов). Для этого решается задача $\Phi\left(\bar{x}_{1}\right)<\Phi\left(\bar{x}_{2}\right)<\ldots<\Phi\left(\bar{x}_{m}\right)$ при $\sum_{j=1}^{4} \lambda_{j}=1$, которая эквивалентна задаче линейного программирования

$$
\begin{gathered}
\lambda_{1}^{\prime}+\lambda_{2}^{\prime}+\lambda_{3}^{\prime}+\lambda_{4}^{\prime} \rightarrow \min , \\
a_{11} \lambda_{1}^{\prime}+a_{12} \lambda_{2}^{\prime}+a_{13} \lambda_{3}^{\prime}+a_{14} \lambda_{4}^{\prime} \leqslant 1, \\
a_{m 1} \lambda_{1}^{\prime}+a_{m 2} \lambda_{2}^{\prime}+a_{m 3} \lambda_{3}^{\prime}+a_{m 4} \lambda^{\prime} \leqslant 1,
\end{gathered}
$$

где $a_{i j}-$ конкретные числа, $\sum_{j=1}^{4} \lambda_{j}^{\prime}=(1 / v) \sum_{j=1}^{4} \lambda_{j}=1 / v$ и $v-$ число, гарантирующее положительность всех элементов $\left\{a_{i j}\right\}$. Искомые $\lambda_{i}$ находятся из выражения $\lambda_{i}=\lambda^{\prime}{ }_{i} / \sum_{i=1}^{4} \lambda_{i}^{\prime}$.

6. Получение оптимального решения исходной задачи оптимизации ТЭБ района путем определения $\min \Phi(\bar{x})$ при $\bar{x}$, удовлетворяющем ограничениям соответствующей многоцелевой задачи.

Однако решение, оптимальное в смысле единого суммарного критерия $Ф$, может и не быть таковым по ряду частных критериев. В этом случае окончательный выбор должны сделать эксперты или лицо, принимающее решение (ЛПР).

Задача агрегации нескольких критериев в один глобальный может решаться итеративным методом. Алгоритм решения включает в себя также шесть этапов: 
I. Сведение критериев $f_{1}-f_{4}$ к безразмерным величинам.

II. Построение области Парето [1 ${ }^{1}$ многоцелевой задачи оптимизации ТЭБ района.

III. Оптимизация критерия $\Phi(\bar{x})$ на области ограничений исходной задачи. Массив $\left\{\lambda_{j}\right\}$, фигурирующий в выражении для функционала $\Phi(\bar{x})$, получается в результате выделения из области Парето решения, наименее удаленного от идеального. Обозначив его через $\bar{x}_{1}$, находим $f\left(\bar{x}_{1}\right)=\left\{f_{j}\left(\bar{x}_{1}\right)\right\}, j=\overline{1,4}$.

IV. Перед ЛПР ставится вопрос - все ли критерии $f_{j}\left(\bar{x}_{1}\right), j=\overline{1,4}$, имеют удовлетворительное значение? [ $\left.{ }^{3}\right]$. ЛПР сравнивает вектор $f\left(\bar{x}_{1}\right)$ с вектором fид. В случае положительного ответа решение окончательно, т. е. вектор $f\left(\bar{x}_{1}\right)$ есть искомый результат. В противном случае ЛПР выделяет критерий, который имеет наименее удовлетворительное значение, и уступку $\Delta f_{j}$, допускающую увеличение значения этого критерия.

$\mathrm{V}$. В область допустимых значений исходной задачи вводится ограничение $f_{j}(\bar{x}) \leqslant f_{j}^{\text {шл }}+\Delta f_{j}$. На полученной области, которую обозначим через $D_{1}$, процедура повторяется вновь, начиная с этапа I. В результате определяем вектор $f\left(\bar{x}_{2}\right)$.

VI. Перед ЛПР ставится вопрос - допустимо ли ухудшение качества какого-либо критерия при переходе от вектора $f\left(\bar{x}_{1}\right)$ к вектору $f\left(\bar{x}_{2}\right)$ ? Если нет, ЛПР изменяет значение $\Delta f_{j}$, и процедура повторяется, начиная с этапа $\mathrm{V}$. Пусть $\Delta f_{j}^{\mathrm{H}}-$ искомое значение уступки и $\bar{x}^{\mathrm{H}}-$ соответствующий ей оптимальный план исходной задачи. Если все значения $f_{i}\left(\bar{x}^{\mathrm{H}}\right), i=\overline{1,4}$, удовлетворяют предпочтениям ЛПР, то процесс заканчивается, в противном случае он повторяется, начиная с этапа IV.

Отметим; что вид обобщенного критерия $\Phi(\bar{x})$ выдает каждому району центральная система. Эта система соблюдает свои интересы при выборе оптимального компромиссного решения из области Парето и при задании критерия такого выбора.

Итак, оптимизация ТЭБ зависит от множества различных факторов развития энергетического хозяйства района. Для учета этих факторов эффективны методы многоцелевого программирования, среди которых в вычислительном отношении просты приближенные методы. Достижение необходимого качества каждого критерия обеспечивается применение́м человеко-машинных процедур. Разработанный метод опробован на ЭВM EC-1022.

\section{ЛИТ Е РА Т У Р А}

1. Я ани м яги К. Э., В сб.: Учет неопределенности исходной информации при оптимизации энергетического хозяйства экономического района, Таллин, Изд. АН ЭССР, 1978 , c. $159-163$.

2. Я анимя ги К. Э., В сб.: Определение перспектив развития энергетического хозяйства экономического района, Таллин, Изд. АН ЭССР, 11977 , с. 171-179.

3. Бена йюн Р., Л ар и чев О. И., М онгольфье Ж., Т ерни Ж., Автоматика и телемеханика, № 8, 108-1115 (1971).

Ннститут термофизики и электрофизики Академии наук Эстонской ССР 